\title{
Differences in the clinical characteristics and outcomes of COVID-19 patients in the epicenter and peripheral areas of the pandemic from China: a retrospective, large-sample, comparative analysis
}

Gang Wang ${ }^{1,2+}$, Feng Ming Luo ${ }^{1,2+}$, Dan Liu ${ }^{1+}$, Jia Sheng Liu ${ }^{3+}$, Ye Wang ${ }^{1}$, Hong Chen ${ }^{4}$, Pan Wen Tian ${ }^{1}$, Tao Fan ${ }^{5}$, Li Tang ${ }^{1}$, He Yu ${ }^{1}$, Lan Wang ${ }^{1}$, Mei Feng ${ }^{1}$, Zhong Ni ${ }^{1}$, Bo Wang ${ }^{1}$, Zhi Fang Song ${ }^{1}$, Xiao Ling Wu ${ }^{1}$, Hong Jun Wang ${ }^{6}$, Xiang Tong ${ }^{1}$, Miao Xue ${ }^{1}$, Xian Ying Lei ${ }^{7}$, Bo Long ${ }^{8}$, Chao Jia ${ }^{9}$, Jun Xiao ${ }^{10}$, Juan Shang ${ }^{11}$, Nian Xiong ${ }^{12,13^{*}+}$, Jian Fei Luo ${ }^{3^{*+}}$, Zong An Liang ${ }^{1 *_{+}}$, Wei Min $\mathrm{Li}^{1^{*+}}$ on behalf of the Sichuan \& Wuhan Collaboration Research Group, and Sichuan Treatment Expert Group for COVID-19, China

\begin{abstract}
Background: There is limited information on the difference in epidemiology, clinical characteristics and outcomes of the initial outbreak of the coronavirus disease (COVID-19) in Wuhan (the epicenter) and Sichuan (the peripheral area) in the early phase of the COVID-19 pandemic. This study was conducted to investigate the differences in the epidemiological and clinical characteristics of patients with COVID-19 between the epicenter and peripheral areas of pandemic and thereby generate information that would be potentially helpful in formulating clinical practice recommendations to tackle the COVID-19 pandemic.
\end{abstract}

(Continued on next page)

\footnotetext{
*Correspondence: weimi003@scu.edu.cn; liang.zongan@163.com; afei099@163.com; nianxiong@hust.edu.cn

${ }^{\dagger}$ Gang Wang, Feng Ming Luo, Dan Liu and Jia Sheng Liu are co-first authors

and contributed equally to this work.

${ }^{+}$Nian Xiong, Jian Fei Luo, Zong An Liang and Wei Min Li are senior authors

and contributed equally to this work.

'Department of Respiratory and Critical Care Medicine, Clinical Research Center for Respiratory Disease, West China Hospital, Sichuan University,

Chengdu 610041, Sichuan, China

${ }^{3}$ Department of Gastrointestinal Surgery, Renmin Hospital of Wuhan

University, Wuhan 430060, Hubei, China

${ }^{12}$ Department of Neurology, Union Hospital, Tongji Medical College,

Huazhong University of Science and Technology, Wuhan 430022, Hubei,

China

Full list of author information is available at the end of the article
}

(c) The Author(s). 2021 Open Access This article is licensed under a Creative Commons Attribution 4.0 International License, which permits use, sharing, adaptation, distribution and reproduction in any medium or format, as long as you give appropriate credit to the original author(s) and the source, provide a link to the Creative Commons licence, and indicate if changes were made. The images or other third party material in this article are included in the article's Creative Commons licence, unless indicated otherwise in a credit line to the material. If material is not included in the article's Creative Commons licence and your intended use is not permitted by statutory regulation or exceeds the permitted use, you will need to obtain permission directly from the copyright holder. To view a copy of this licence, visit http://creativecommons.org/licenses/by/4.0/. The Creative Commons Public Domain Dedication waiver (http://creativecommons.org/publicdomain/zero/1.0/) applies to the data made available in this article, unless otherwise stated in a credit line to the data. 
(Continued from previous page)

Methods: The Sichuan \& Wuhan Collaboration Research Group for COVID-19 established two retrospective cohorts that separately reflect the epicenter and peripheral area during the early pandemic. The epidemiology, clinical characteristics and outcomes of patients in the two groups were compared. Multivariate regression analyses were used to estimate the adjusted odds ratios (aOR) with regard to the outcomes.

Results: The Wuhan (epicenter) cohort included 710 randomly selected patients, and the peripheral (Sichuan) cohort included 474 consecutive patients. A higher proportion of patients from the periphery had upper airway symptoms, whereas a lower proportion of patients in the epicenter had lower airway symptoms and comorbidities. Patients in the epicenter had a higher risk of death ( $\mathrm{aOR}=7.64)$, intensive care unit (ICU) admission (aOR=1.66), delayed time from illness onset to hospital and ICU admission (aOR=6.29 and aOR=8.03, respectively), and prolonged duration of viral shedding (aOR=1.64).

Conclusions: The worse outcomes in the epicenter could be explained by the prolonged time from illness onset to hospital and ICU admission. This could potentially have been associated with elevated systemic inflammation secondary to organ dysfunction and prolonged duration of virus shedding independent of age and comorbidities. Thus, early supportive care could achieve better clinical outcomes.

Keywords: COVID-19, Case fatality, Epicenter, Peripheral area, Pandemic, Comparative analysis

\section{Background}

In December 2019, an outbreak of pneumonia of unknown cause was identified in Wuhan, the capital of Hubei province in China. A novel coronavirus, the severe acute respiratory syndrome coronavirus 2 (SARSCoV-2), which had not been detected previously in humans, was identified subsequently by Chinese scientists as the cause [1]. The disease was named the coronavirus disease 2019 (COVID-19) by the World Health Organization (WHO). The clinical spectrum of COVID19 appears to be wide, and ranges from self-limited mild upper respiratory tract illness to severe pneumonia causing hospitalization or death. The clinical characteristics of some COVID-19 case series in Wuhan, the epicenter of the pandemic, have been previously reported in detail. The reports indicated that 26 to $33 \%$ of patients required intensive care and 4 to $15 \%$ died [2-4].

After the outbreak of COVID-19 in Wuhan, the government of the Sichuan province implemented strict measures to combat COVID-19. The Health Commission of Sichuan Province (HCSP) focused on traditional public health outbreak response tactics, including isolation, quarantine, social distancing, and community containment, as recommended by the National Health Commission of China. All medical resources were allocated by the HCSP to ensure efficient use. An expert panel drawn from multidisciplinary teams was established and comprised 125 physicians who were led by Dr. Wei Min Li and Dr. Zong An Liang (the corresponding authors of this study) since January 15, 2020. This expert panel soon released emergency prevention and control guidelines for COVID-19 in the medical institutions of the Sichuan province [5]. Furthermore, we funded two additional important expert panels with psychological counseling [6] and traditional Chinese medicine as complementary and alternative treatment options [7, 8]. Physicians caring for severely or critically ill patients could receive daily internet consultations with members of the expert panel. There were 208 designated hospitals across Sichuan Province that were accessible for SARS-CoV-2-suspected or -confirmed individuals. This arrangement resulted in improved outcomes in Sichuan province, one of the peripheral areas of the pandemic. In other peripheral areas, 2 to $10.1 \%$ of patients needing intensive care, and an approximately $1.0 \%$ mortality rate were reported in recently published studies [9-11].

The factors underlying the significantly different clinical outcomes between the epicenter and peripheral areas affected by the pandemic remains largely unexplored. Recently, Liang et al. [12] observed the clinical characteristics and outcomes of hospitalized patients with COVID19who were treated in Hubei (epicenter) or outside Hubei (non-epicenter). However, as theirs is a multicenter study, the possibility of selection bias for the included patients cannot be ruled out. Furthermore, hospitalized patients in Hubei but not in Wuhan, would not be well representative of the first-generation COVID-19 cases. Considering the rapidly increasing number of cases with SARS-CoV-2 infection worldwide, the existing research into the differences between the epicenter and peripheral areas of the pandemic in the clinical characteristics and outcomes of COVID-19 patients was insufficient.

This study could provide information that would be potentially helpful in formulating clinical practice recommendations to tackle the COVID-19 pandemic worldwide.

\section{Methods}

\section{Study design and subjects}

This was a retrospective study based on two cohorts evaluated by the Sichuan and Wuhan Collaboration Research Group for COVID-19, China. The Wuhan cohort, 
drawn from the epicenter area of the pandemic, was formed using a computer-generated simple random sampling method that was applied to enroll subjects from two designated hospitals, namely the Wuhan Red Cross Hospital and Renmin Hospital of Wuhan University, Wuhan, China. The Sichuan cohort, as the group of patients from the peripheral area of the pandemic, consisted of SARS-CoV-2-confirmed patients who were consecutively recruited from 41 designated hospitals until March 12, 2020. Based on the exposure history, we further divided the Sichuan cohort into two sub-cohorts, with or without Wuhan exposure history. All patients enrolled in this study were diagnosed with COVID-19 according to the interim guidance issued by the National Health Commission of China and the WHO [13]. SARS$\mathrm{CoV}-2$ infection was confirmed by a positive result on a real-time reverse-transcriptase-polymerase-chain-reaction of nasopharyngeal, pharyngeal, throat-swab or sputum specimens. Some of these patients were included in studies reported by Wei et al. [14], Xiong et al. [15] and Xiong et al. [16]; however, their study purposes are significantly different from those of this study.

\section{Data collection}

The medical records of patients with COVID-19 were reviewed by members of the trained research team. Epidemiological, demographic, clinical, laboratory, radiological, treatment and outcome data were collected by using standardized data collection forms (modified case record form for the clinical characterization of severe acute respiratory infection that was shared by the International Severe Acute Respiratory and Emerging Infection Consortium [ISARIC]) from the electronic medical records. The cutoff date was Mar 12, 2020. We collected details of the exposure history, clinical symptoms and signs, and laboratory findings on admission. Laboratory examinations were performed according to the clinical care needs of the patients. Data on radiological abnormalities were extracted from the selected documentation. Patients were excluded if their medical records were not available. A team of trained researchers abstracted the data and entered the structured spreadsheet. All data were cross-checked.

\section{Study outcomes}

The primary outcomes included death or mechanical ventilation whether or not it involved intensive care unit (ICU) admission. Mechanical ventilation was performed in the ward if ICU admission was not possible due to the overwhelming numbers of COVID-19 patients. The secondary outcomes were the rate of ICU admission, time from illness onset to ICU admission and discharge, length of hospital stay, and duration of viral shedding after COVID-19 onset. Duration of viral shedding was defined to ended when two consecutive negative results with qPCR detection were obtained at time intervals greater than $24 \mathrm{~h}$. The criteria for discharge were absence of fever for at least 3 days, substantial improvement in both lungs on chest computed tomography (CT), clinical remission of respiratory symptoms and comorbidities, and cessation of SARS-Cov-2.

\section{Statistical analysis}

Continuous variables were compared using the Student's $t$ test or the Mann-Whitney $U$ test; categorical variables were compared by the chi-square test or Fisher's exact test as appropriate. Logistic or linear regression was performed to identify clinical variables that were associated with outcomes. The detailed statistical analysis is described in the supplementary data.

\section{Results \\ Epidemiological and clinical characteristics at hospitalization}

As of March 12, 2020, a total of 1979 cases from the two hospitals in Wuhan were identified. The Wuhan cohort included $35.9 \%(n=710)$ of all patients from Wuhan, selected using a computer-generated simple random sampling method, formed the Wuhan cohort. In the Wuhan cohort, illness onset in the first case was noted on December 24, 2019, and the first hospitalization occurred on January 5, 2020 (Fig. 1a).

There were 538 patients with COVID-19 who were consecutively admitted to 41 designated hospitals in $\mathrm{Si}$ chuan Province. The Sichuan cohort comprised 474 patients (Fig. 1b), when 64 patients with inaccessible medical records were excluded. Epidemiological data indicated that the first cases of SARS-CoV-2 infection in the Sichuan cohort occurred in December 31, 2019, and the first case was admitted to the designated hospital on January 16, 2020 (Fig. 1c). The daily Wuhan-related exposure cases with onset of COVID-19 in the Sichuan cohort peaked on January 23, 2020, and those without Wuhan-related exposure peaked on February 1, 2020 (Fig. 2a and b). The median time from illness onset to admission in the Sichuan cohort was significantly shorter than that in the Wuhan cohort $(5.0[2.0,9.0]$ vs. $10.0[7.0$, $15.0]$ days, $P<0.001)$. The Sichuan cohort had a lower proportion of patients with an exposure history than that in the Wuhan cohort (64.3\% vs. $99.3 \%, P<0.001)$.

The demographic and clinical characteristics of these patients are shown in Table 1 and S1. Patients in the Sichuan cohort were younger $(44[32.0,54.0]$ vs. 58 [43.0, $67.0]$ yrs., $P<0.001)$, there were fewer females $(46.4 \%$ vs. $54.1 \%, P=0.010$ ), and included a higher number of current smokers $(14.5 \%$ vs. $5.1 \%, P<0.001)$. Two patients $(0.4 \%)$ in the Sichuan cohort and $13(1.8 \%)$ in the $\mathrm{Wu}$ han cohort were healthcare workers $(P=0.033)$. The 


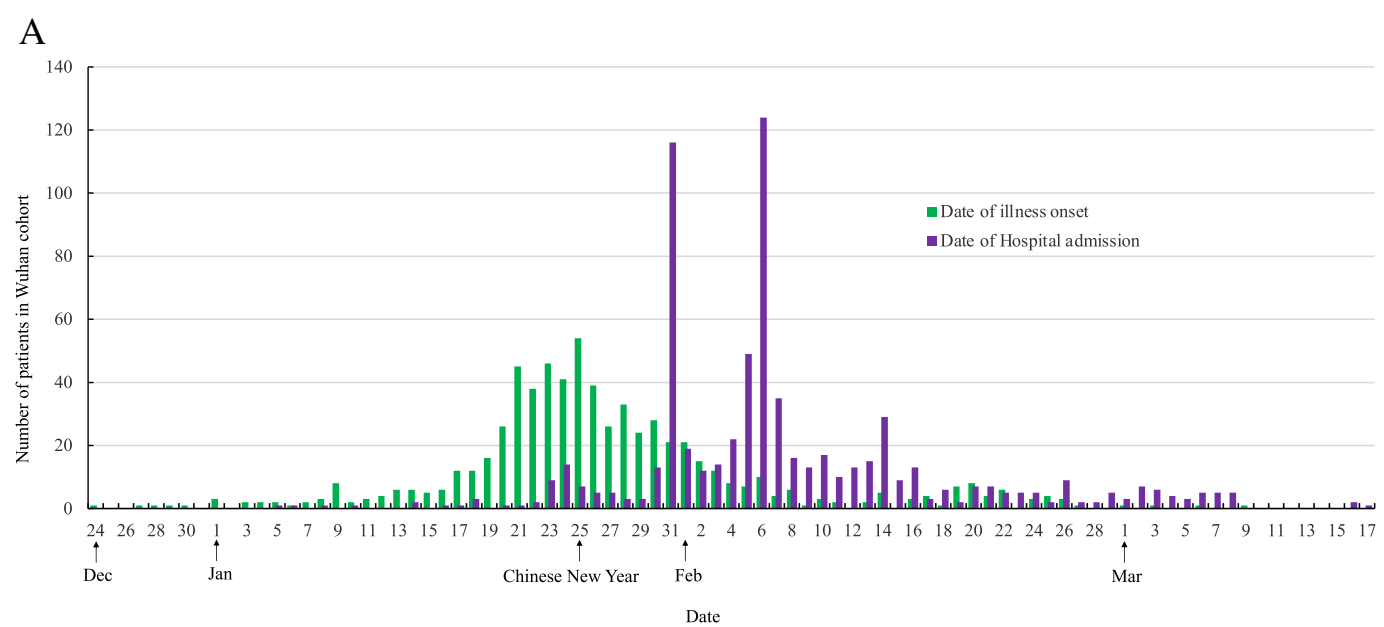

B
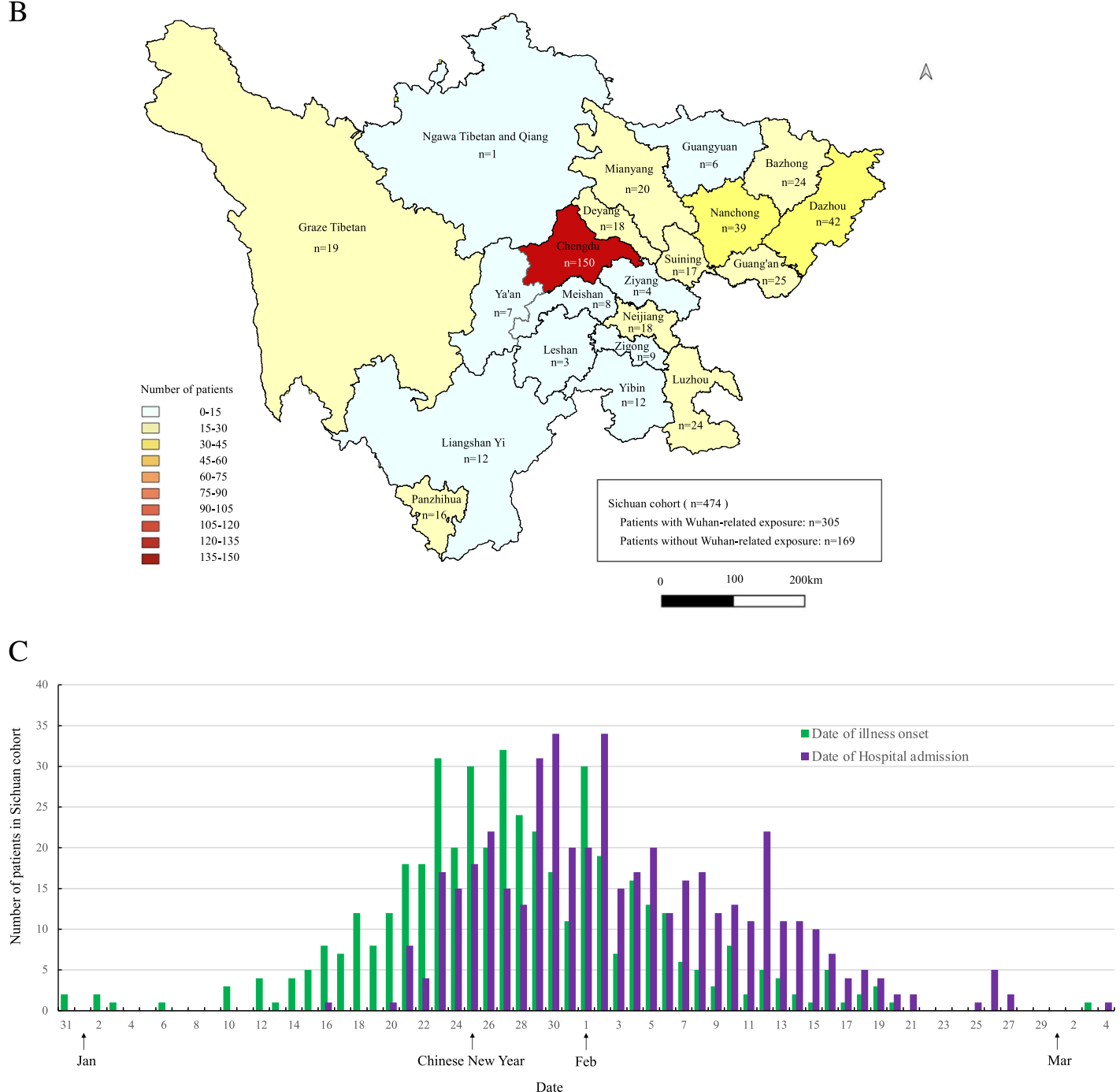

Fig. 1 (See legend on next page.) 
(See figure on previous page.)

Fig. 1 a Time of illness onset and hospital admission of patients in the Wuhan cohort; b Distribution of patients with COVID-19 in the Sichuan cohort (The data of administrative areas were downloaded from the Database of Global Administrative Areas [GADM] freely available for academic use and we drew this figure using QGIS software version 3.8.3); c Time of illness onset and hospital admission of patients in the Sichuan cohort

commonest comorbidity in both cohorts was hypertension (23.6\%), followed by diabetes (11.9\%). The Wuhan cohort had more cases with comorbidities $(51.2 \%$ vs. $43.8 \%, P=0.012)$ as assessed by the Charlson Comorbidity Index [17] (CCI) $(2.0[2.0,3]$ vs. $0[0,1.0], P<0.001)$. Fewer patients in the Sichuan cohort had a history of coronary heart disease $(P=0.004)$, liver disease $(P<0.001)$, stroke $(P=0.026)$, hypertension $(P<0.001)$, and malignancy $(P=$ $0.012)$ than those in the Wuhan cohort.

Fever was the commonest symptom and was present in $61.8 \%$ of patients in the Sichuan cohort or $65.1 \%$ of patients in the Wuhan cohort, but the difference was not significant $(P=0.246)$. The Sichuan cohort had a higher incidence of productive cough than the Wuhan cohort $(P=0.012)$. However, the Wuhan cohort seemed to have a higher symptomatic burden with regard to the lower respiratory tract, including shortness of breath (25.4\% vs. $9.0 \%, P<0.001)$, chest distress $(23.8 \%$ vs. $9.0 \%$, $P<0.001)$, wheeze $(13.9 \%$ vs. $4.8 \%, P<0.001)$, and general symptomatic burden, including fatigue $(36.2 \%$ vs. $22.3 \%$, $P<0.001$ ), hemoptysis ( $3.0 \%$ vs. $1.1 \%, P=0.028)$, altered consciousness $(1.8 \%$ vs. $0.2 \%, P=0.011)$, and diarrhea $(12.1 \%$ vs. $6.3 \%, P=0.001)$. In contrast, the Sichuan cohort was more likely to have upper respiratory symptoms, including pharyngalgia $(13.9 \%$ vs. $7.5 \%, P<0.001)$, rhinorrhea $(5.0 \%$ vs. $1.4 \%, P<0.001)$, nasal obstruction ( $3.4 \%$ vs. $1.1 \%, P=0.007)$, and headache $(10.1 \%$ vs. $4.6 \%$, $P<0.001$ ) (Fig. 3a). Different severity distributions were observed between the two cohorts $(P<0.001)$, as assessed by CURB-65 and MuLBSTA (both $P<0.001$ ). More than $75 \%$ of patients in both cohorts had mild or general disease, although the Sichuan cohort had a higher proportion of severe cases $(17.0 \%$ vs. $8.4 \%)$ and the Wuhan cohort had more critically ill patients $(13.6 \%$ vs. $6.3 \%)$. Chest CT radiographs in the Wuhan cohort were more likely to show bilateral lung involvement $(P=0.012)$ and consolidation $(P=0.006)$.

There was no difference in white blood cell count, lymphocyte count, prothrombin time, albumin, alanine aminotransferase, aspartate aminotransferase, procalcitonin and interleukin 6 (IL-6) between the two cohorts. The Sichuan cohort had lower neutrophil count $(P<$ $0.001)$, platelet count $(P<0.001)$, D-dimer levels $(P=$ $0.001)$, and $C$-reactive protein levels $(P<0.001)$ and higher levels of hemoglobin $(P=0.015)$, activated partial thromboplastin time $(P<0.001)$, creatinine $(P=0.040)$, and creatine kinase $(P<0.001)$ (Table 1$)$.

\section{Treatments and clinical outcomes}

A comparison of treatments and clinical outcomes between the two cohorts is shown in Table 2. Almost all patients received antiviral treatment in Sichuan (94.7\%) or Wuhan (93.2\%). Fewer patients in the Sichuan cohort received antibiotics $(P<0.001)$, corticosteroids $(P<0.001)$ and supplemental oxygen therapy $(P<0.001)$.

The case fatality rate in the Sichuan cohort was obviously lower than that in the Wuhan cohort $(0.6 \%$ vs. $8.3 \%, P<0.001)$. However, there was no significant difference in the proportion of patients receiving noninvasive mechanical ventilation or invasive mechanical ventilation between the two cohorts $(5.7 \%$ vs. $5.9 \%, P=0.872$ and $1.7 \%$ vs. $1.4 \%, P=0.701)$. With regard to the secondary outcomes, the proportion of patients who were admitted to the ICU in the Sichuan cohort was significantly lower than that in the Wuhan cohort $(6.3 \%$ vs. $13.6 \%, P<$ 0.001). The time from illness onset to ICU admission and time from illness onset to discharge in the Sichuan cohort were shorter than that in the Wuhan cohort $(7.0$ $[4.0,10.5]$ vs. $11.5[8.8,24.3]$ days, $P<0.001$ and 23.0 $[18.0,31.0]$ vs. $28.0[18.0,38.0]$ days, $P<0.001)$. The length of hospital stay in the Sichuan cohort was much longer than that in the Wuhan cohort $(17.0[12.0,24.0]$ vs. $14.0[9.0,24.0]$ days, $P<0.001)$. In contrast, the Wuhan cohort had a significantly prolonged duration of SARS-CoV-2 shedding than that in the Sichuan cohort $(19.0[13.0,28.0]$ vs. $14.0[10.0,19.0]$ days, $P<0.001)$.

\section{Logistic regression analyses}

Multivariable logistic regression models were used to explore the differences in clinical outcomes between the Sichuan and Wuhan cohorts (Table 3). The results showed that the Wuhan cohort had higher risk of death $(\mathrm{aOR}=7.64,95 \% \mathrm{CI}=[2.31,25.27], P=0.001)$, ICU admission $(\mathrm{aOR}=1.66,95 \% \mathrm{CI}=[1.05,2.63], P=0.031)$, delayed time from illness onset to hospital $(\mathrm{aOR}=6.29,95 \% \mathrm{CI}=$ $[4.70,8.40], P<0.001)$ and ICU admission $(\mathrm{aOR}=8.03$, $95 \% \mathrm{CI}=[1.74,37.06], \quad P<0.001)$ admissions, prolonged duration of viral shedding after COVID-19 onset (aOR= 1.64, $95 \% \mathrm{CI}=[1.15,2.33], P=0.006)$, a decreased hospital stay $(\mathrm{aOR}=0.41,95 \% \mathrm{CI}=[0.32,0.53], \quad P<0.001)$ after adjusting for age, sex, smoking status and the CCI. There was no difference in time from illness onset to discharge (aOR=0.99, 95\% $\mathrm{CI}=[0.77,1.28], P=0.968)$ after adjusting for these confounders. When we additionally 

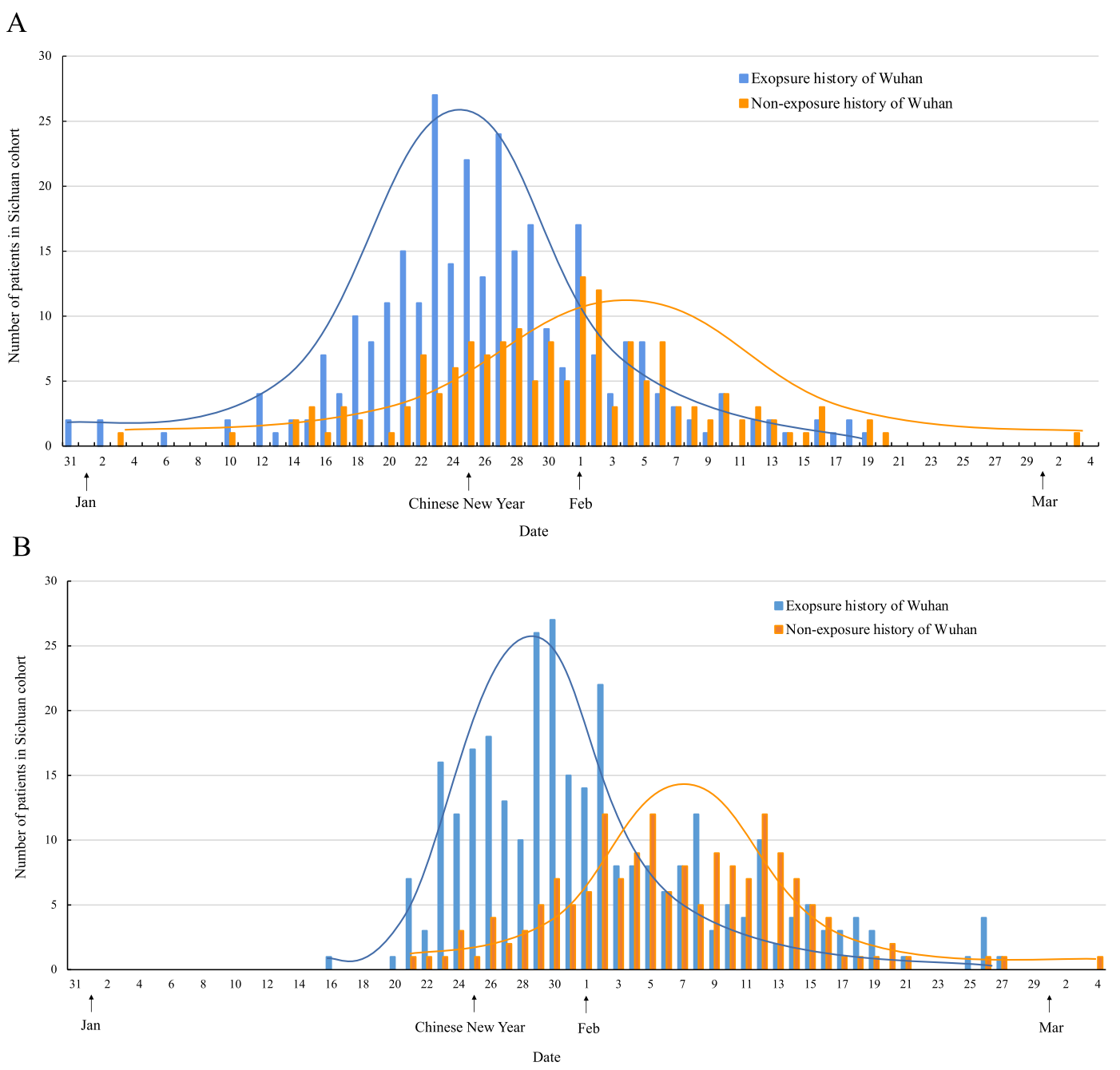

$\mathrm{C}$

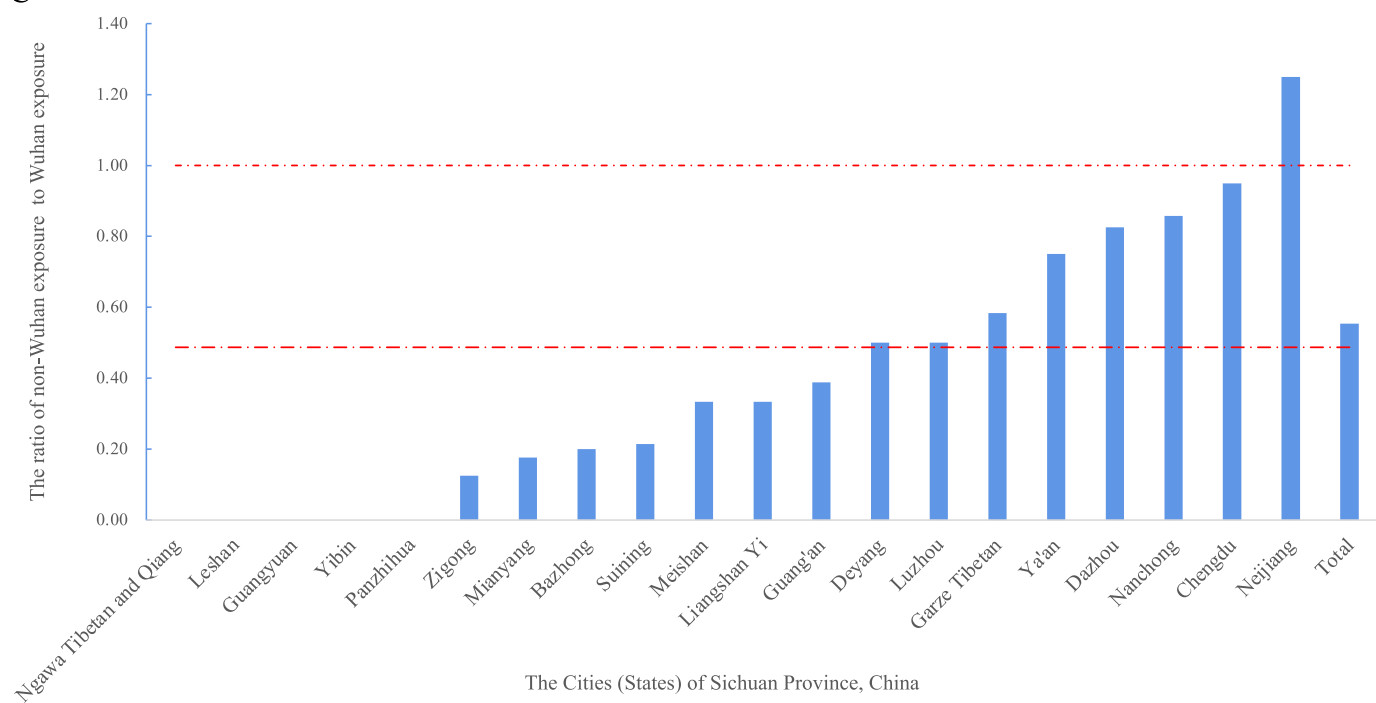

Fig. 2 a Time of illness onset of patients with or without Wuhan-related exposure in the Sichuan cohort; $\mathbf{b}$ Time of hospital admission of patient with or without Wuhan-related exposure in the Sichuan cohort; $\mathbf{c}$ The ratio of the number of patients without Wuhan-related exposure to cases with Wuhan exposure in the Sichuan cohort 
Table 1 Demographics and clinical characteristics of patients in the Sichuan and Wuhan cohorts

\begin{tabular}{|c|c|c|c|c|c|}
\hline Variable & Total & Sichuan cohort & Wuhan cohort & $x^{2} / Z$ & $P$ value \\
\hline n & 1184 & 474 & 710 & & \\
\hline Female, n (\%) & $604(51.0)$ & $220(46.4)$ & $384(54.1)$ & 6.693 & 0.010 \\
\hline Age, years (Median [IQR]) & $50.50(37.00,64.00)$ & $44.00(32.00,54.00)$ & $58.00(43.00,67.00)$ & 12.054 & $<0.001$ \\
\hline Travel in Wuhan/residence in Wuhan/no exposure history, $\mathrm{n}$ & $141 / 869 / 174$ & $128 / 177 / 169$ & $13 / 692 / 5$ & 527.493 & $<0.001$ \\
\hline Health care workers, n (\%) & $15(1.3)$ & $2(0.4)$ & $13(1.8)$ & 4.532 & 0.033 \\
\hline Current/ ever/ never smoking, $\mathrm{n}$ & $96 / 42 / 892$ & $67 / 18 / 376$ & $29 / 24 / 516$ & 26.843 & $<0.001$ \\
\hline Any comorbidity & $574(48.2)$ & $208(43.8)$ & $366(51.2)$ & 6.258 & 0.012 \\
\hline Charlson Comorbidity Index & $1.0(0-2.0)$ & $0(0-1.0)$ & $2(2.0-3.0)$ & -9.190 & $<0.001$ \\
\hline Disease severity status, $\mathrm{n}$ & & & & 97.524 & $<0.001$ \\
\hline Mild/general/severe/critical & 194/725/141/127 & 28/337/81/30 & 166/388/60/97 & & \\
\hline \multicolumn{6}{|l|}{ CURB-65 score, n (\%) } \\
\hline $0-1 / 2 / 3-5$ & $837 / 68 / 20$ & $369 / 12 / 2$ & $468 / 56 / 18$ & 26.430 & $<0.001$ \\
\hline MuLBSTA score (Median [IQR]) & $7.00(5.00,9.00)$ & $5.00(5.00,9.00)$ & $7.00(5.00,9.00)$ & 3.96 & $<0.001$ \\
\hline \multicolumn{6}{|l|}{ Laboratory findings, Median (IQR) } \\
\hline White blood cell count, $\times 10^{9} / \mathrm{L}$ & $5.45(4.22,7.07)$ & $5.37(4.18,5.37)$ & $5.58(4.22,7.28)$ & 1.570 & 0.088 \\
\hline Neutrophil count, $\times 10^{9} / \mathrm{L}$ & $3.45(2.50,4.99)$ & $3.45(2.53,4.73)$ & $3.45(2.47,5.26)$ & 2.723 & $<0.001$ \\
\hline Lymphocyte count, $\times 10^{9} / \mathrm{L}$ & $1.18(0.83,1.63)$ & $1.18(0.81,1.60)$ & $1.18(0.84,1.64)$ & 1.074 & 0.157 \\
\hline Eosinophil count, $\times 10^{9} / \mathrm{L}$ & $0.30(0.00,1.30)$ & $0.20(0.01,0.80)$ & $0.50(0.0,1.7)$ & 5.751 & $<0.001$ \\
\hline Hemoglobin, $g / L$ & $131.00(119.00,144.00)$ & $137.00(126.00,151.00)$ & $127.00(117.00,137.00)$ & 8.049 & 0.015 \\
\hline Platelet count, $\times 10^{9} / \mathrm{L}$ & $197.00(148.00,262.50)$ & $175.00(137.00,230.50)$ & $215.00(165.00,281.00)$ & 7.909 & $<0.001$ \\
\hline Activated partial thromboplastin time, s & $28.50(25.90,32.20)$ & $30.90(27.70,34.90)$ & $27.40(25.20,29.90)$ & 10.129 & $<0.001$ \\
\hline Prothrombin time, s & $12.20(11.50,13.00)$ & $12.60(11.70,13.30)$ & $12.00(11.33,12.70)$ & 2.052 & 0.115 \\
\hline D-dimer, mg/L & $0.56(0.29,1.59)$ & $0.50(0.22,1.17)$ & $0.63(0.33,1.79)$ & 3.194 & 0.001 \\
\hline Albumin, $g / L$ & $39.70(35.50,43.30)$ & $43.00(39.60,45.70)$ & $37.70(34.00,40.80)$ & 11.556 & 0.128 \\
\hline Creatinine, $\mu \mathrm{mol} / \mathrm{L}$ & $63.00(51.00,76.15)$ & $65.20(53.00,77.33)$ & $62.00(50.70,75.00)$ & 2.051 & 0.040 \\
\hline Creatine kinase, U/L & $62.70(41.00,106.25)$ & $71.00(50.00,122.00)$ & $56.00(34.40,99.00)$ & 5.181 & $<0.001$ \\
\hline Alanine aminotransferase, $\mathrm{U} / \mathrm{L}$ & $23.55(16.00,39.05)$ & $24.00(16.00,39.90)$ & $23.00(15.48,39.00)$ & 0.433 & 0.665 \\
\hline Aspartate aminotransferase, U/L & $25.00(19.67,35.73)$ & $25.60(20.00,35.00)$ & $25.00(19.00,36.00)$ & 0.459 & 0.647 \\
\hline C-reactive protein, mg/L & $19.95(6.80,53.92)$ & $10.16(2.67,24.72)$ & $28.45(8.70,65.20)$ & 6.066 & $<0.001$ \\
\hline Procalcitonin, ng/mL & $0.05(0.04,0.12)$ & $0.06(0.04,0.17)$ & $0.05(0.03,0.11)$ & 2.06 & 0.039 \\
\hline Hypersensitive troponin I, pg/ml & $0.01(0.01,0.01)$ & $0.01(0.01,0.01)$ & $0.02(0.01,0.05)$ & 3.615 & $<0.001$ \\
\hline \multicolumn{6}{|l|}{ Chest CT, n (\%) } \\
\hline Bilateral lungs involvement & $741(93.1)$ & $328(90.6)$ & $413(95.2)$ & 6.363 & 0.012 \\
\hline Consolidation & $180(19.6)$ & $73(16.0)$ & $107(23.2)$ & 7.533 & 0.006 \\
\hline Ground-glass opacity & $666(71.3)$ & $328(71.6)$ & $338(71.0)$ & 0.042 & 0.837 \\
\hline Linear opacity & $257(27.8)$ & $112(24.5)$ & $145(31.2)$ & 5.200 & 0.023 \\
\hline Pleural effusion & $49(5.4)$ & $19(4.2)$ & $30(6.6)$ & 2.559 & 0.110 \\
\hline
\end{tabular}

CT Computed tomography

adjusted for time from illness onset to hospitalization, the risk of the Wuhan cohort was nearly unchanged; however, the Sichuan cohort had a lower risk for extended time from illness onset to discharge $(\mathrm{aOR}=0.46$, 95\% $\mathrm{CI}=[0.34,0.63], P<0.001)$.

In the overall study population of COVID-19 patients from the two cohorts, we constructed multivariable logistic regression models to detect the risk factors at admission for death, ICU admission, mechanical ventilation and duration of viral shedding after COVID-19 onset (Table S2). After adjusting for the cohort sites, sex, age, smoking status and the CCI, we found that white blood cells $\left(>10 \times 10^{9} / \mathrm{L}\right)$, neutrophils $\left(>6.3 \times 10^{9} / \mathrm{L}\right)$, lymphocytes $\left(>1.0 \times 10^{9} / \mathrm{L}\right)$, hemoglobin $(<90 \mathrm{~g} / \mathrm{L})$, D-dimer $(>$ $0.5 \mathrm{mg} / \mathrm{L}$ ), creatine kinase (>185 IU/L), hyper-sensitive troponin 


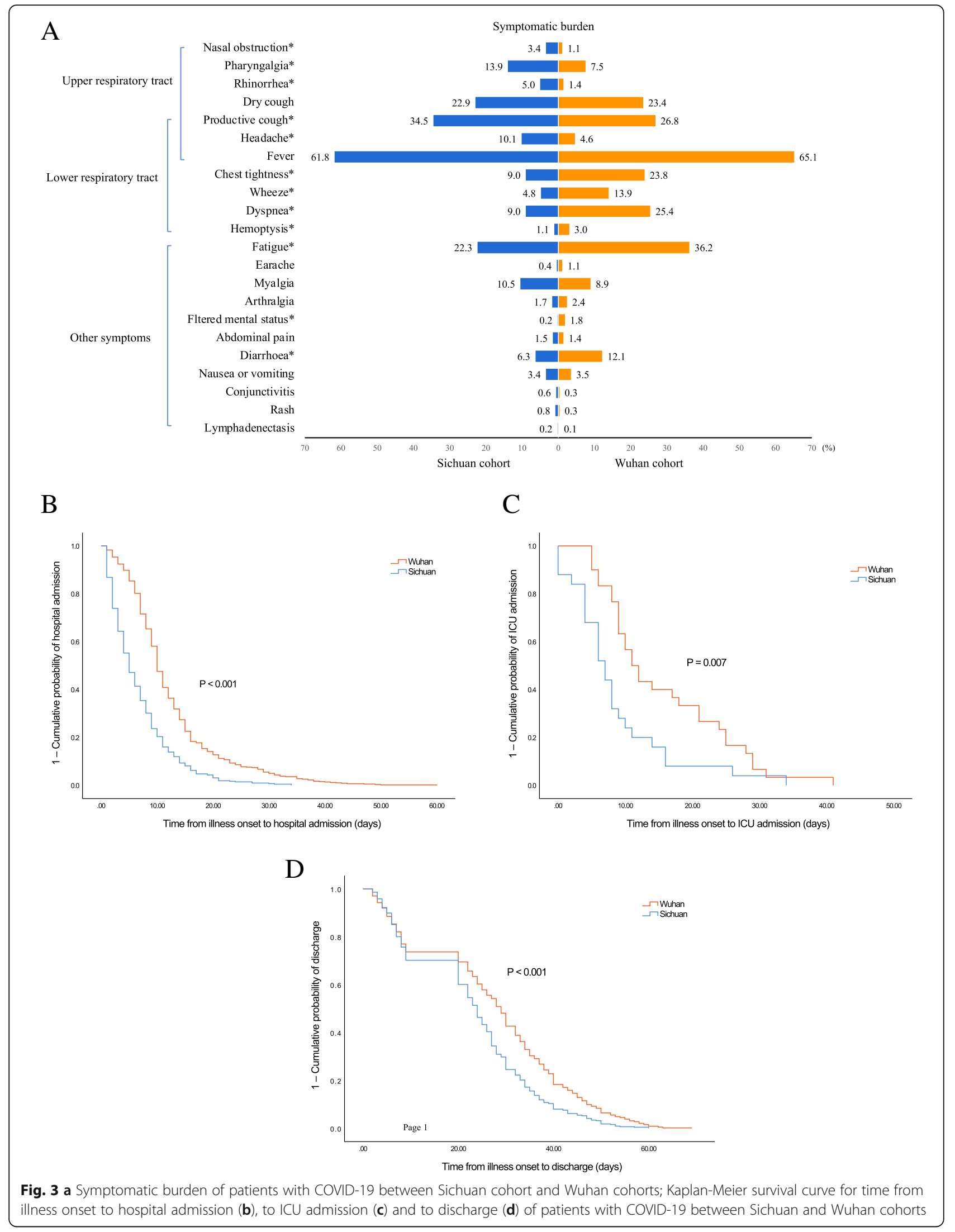


Table 2 Treatments and outcomes of the patients in the Sichuan and Wuhan cohorts

\begin{tabular}{|c|c|c|c|c|c|}
\hline Variable & Total & Sichuan cohort & Wuhan cohort & $x^{2} / Z$ & $P$ value \\
\hline$n$ & 1184 & 474 & 710 & & \\
\hline \multicolumn{6}{|l|}{ Treatments, n (\%) } \\
\hline Antiviral treatment & $1110(93.8)$ & $448(94.7)$ & $662(93.2)$ & 1.067 & 0.302 \\
\hline Antibiotics & $698(59.0)$ & $204(43.0)$ & $494(69.6)$ & 82.734 & $<0.001$ \\
\hline Antifungal treatment & $35(3.0)$ & $15(3.2)$ & $20(2.8)$ & 0.120 & 0.729 \\
\hline Corticosteroids & $272(23.0)$ & $63(13.3)$ & $209(29.4)$ & 41.872 & $<0.001$ \\
\hline Intravenous immunoglobin & $9(0.8)$ & $3(0.6)$ & $6(0.8)$ & 0.170 & 0.680 \\
\hline Oxygen therapy & $791(66.9)$ & $273(57.7)$ & $518(731)$ & 30.175 & $<0.001$ \\
\hline Prone-position ventilation & $30(2.5)$ & $22(4.6)$ & $8(1.1)$ & 14.217 & $<0.001$ \\
\hline Tracheotomy & $8(0.7)$ & $4(0.8)$ & $4(0.6)$ & & $0.720^{*}$ \\
\hline ECMO & $3(0.3)$ & $1(0.2)$ & $2(0.3)$ & & $1.000^{*}$ \\
\hline Renal replacement & $9(0.8)$ & $5(1.1)$ & $4(0.6)$ & & $0.497^{*}$ \\
\hline Blood transfusion & $165(14.0)$ & $30(6.3)$ & $135(19.1)$ & 38.587 & $<0.001$ \\
\hline Nutrition support & $131(11.1)$ & $52(11.0)$ & $79(11.2)$ & 0.014 & 0.906 \\
\hline TCM treatments & $912(77.0)$ & $418(88.2)$ & $494(69.6)$ & 55.620 & $<0.001$ \\
\hline Physiotherapy & $29(2.4)$ & $24(5.1)$ & $5(0.7)$ & 22.605 & $<0.001$ \\
\hline \multicolumn{6}{|l|}{ Outcomes } \\
\hline Death, n (\%) & $62(5.2)$ & $3(0.6)$ & $59(8.3)$ & 33.758 & $<0.001$ \\
\hline ICU admission, n (\%) & $127(10.7)$ & $30(6.3)$ & $97(13.7)$ & 15.961 & $<0.001$ \\
\hline Noninvasive mechanical ventilation, n (\%) & $69(5.8)$ & $27(5.7)$ & $42(5.9)$ & 0.025 & 0.875 \\
\hline Invasive mechanical ventilation, n (\%) & $18(1.5)$ & $8(1.7)$ & $10(1.4)$ & 0.148 & 0.700 \\
\hline Time from illness onset to hospitalization, days & $8.00(4.00,13.00)$ & $5.00(2.00,9.00)$ & $10.00(7.00,15.00)$ & 13.626 & $<0.001$ \\
\hline Hospital stay, days & $16.00(9.00,24.00)$ & $17.00(12.00,24.00)$ & $14.00(9.00,24.00)$ & -2.726 & $<0.001$ \\
\hline Time from illness onset to ICU admission, days & $9.00(6.00,17.00)$ & $7.00(4.00,10.50)$ & $11.50(8.75,24.25)$ & $3.192^{\mathrm{a}}$ & $<0.001$ \\
\hline Time from hospitalization to ICU admission, days & $3.00(0.00,9.00)$ & $4.00(0.00,9.00)$ & $3.00(0.00,10.50)$ & 0.415 & 0.678 \\
\hline Time from illness onset to discharge, days & $26.00(18.00,35.00)$ & $23.00(18.00,31.00)$ & $28.00(18.00,38.00)$ & 5.693 & $<0.001$ \\
\hline Time from illness onset to death, days & $16.50(13.00,21.75)$ & $13.00(11,-)$ & $17.00(13.00,23.50)$ & $1.240^{\mathrm{a}}$ & 0.235 \\
\hline Time from hospital admission to death, days & $5.00(3.00,7.00)$ & $10.00(6.00,-)$ & $4.00(3.00,7.00)$ & 1.427 & 0.153 \\
\hline Duration of viral shedding, days & $14.00(9.00,22.00)$ & $13.00(8.00,18.00)$ & $17.00(11.00,27.00)$ & 6.665 & $<0.001$ \\
\hline
\end{tabular}

* Fisher's exact test

ECMO Extracorporeal membrane oxygenation, TCM Traditional Chinese medicine

I ( $>0.04 \mathrm{ng} / \mathrm{mL})$, alanine aminotransferase (> $50 \mathrm{IU} / \mathrm{L})$, aspartate aminotransferase $(>40 \mathrm{IU} / \mathrm{L})$, procalcitonin $(>0.5 \mathrm{ng} / \mathrm{mL})$ and delayed hospitalization were associated with death, ICU admission and mechanical ventilation. In addition, we found that time from illness onset to hospitalization was associated with prolonged duration of virus shedding (adjusted $\beta=0.11,95 \% \mathrm{CI}=$ $[0.03,0.24], P=0.009)$.

We analyzed the relationship between the delay in hospitalization and elevated systemic inflammation and features of organ dysfunction. The time from illness onset to hospitalization was positively correlated with systemic inflammatory cells such as white blood cells $(\mathrm{r}=0.086, P=$ 0.004), neutrophils ( $\mathrm{r}=0.089, P=0.003)$, eosinophils $(\mathrm{r}=0.116$, $P<0.001)$, platelets $(\mathrm{r}=0.212, P<0.001)$, and inflammatory biomarkers, such as D-dimer $(\mathrm{r}=0.101, \quad P=0.004)$, procalcitonin ( $\mathrm{r}=-0.093, P=0.019)$, features of organ dysfunction, such as hemoglobin $(\mathrm{r}=-0.155, P<0.001), \mathrm{BUN}(\mathrm{r}=$ $0.10, P=0.002)$, creatine $(\mathrm{r}=-0.094, P=0.003)$, albumin $(\mathrm{r}=-$ 0.263, $P<0.001$ ), and APTT $(r=-0.247, P<0.001)$ (Table 4). After adjusting for age, the correlations of delay in hospitalization with BUN and D-dimer did not achieve statistical significance, which indicated that the delay in hospitalization was independent of age. We further analyzed the relationship between the delay in hospitalization and ICU admission with elevated systemic inflammation after adjusting for age, sex, smoking, and steroid use. In general, the relationship between the delay in hospitalization and elevated systemic inflammation did not change, which implied that these relationships were independent of age, sex, smoking, and steroid use. 
Table 3 Risk of adverse outcomes in Wuhan cohort in reference to the Sichuan cohort

\begin{tabular}{|c|c|c|c|c|c|c|}
\hline \multirow[t]{2}{*}{ Outcomes } & \multicolumn{3}{|c|}{ Unadjusted } & \multicolumn{3}{|c|}{ Adjusted $^{a}$} \\
\hline & OR & $95 \% \mathrm{Cl}$ & $P$ & OR & $95 \% \mathrm{Cl}$ & $P$ \\
\hline Death & 14.286 & $4.444-45.455$ & $<0.001$ & 7.643 & $2.311-25.274$ & 0.001 \\
\hline ICU admission & 2.347 & $1.531-3.597$ & $<0.001$ & 1.659 & $1.047-2.627$ & 0.031 \\
\hline Non-invasive mechanical ventilation & 1.044 & $0.635-1.718$ & 0.865 & 0.651 & $0.376-1.127$ & 0.125 \\
\hline Invasive mechanical ventilation & 0.835 & $0.327-2.132$ & 0.705 & 0.381 & $0.138-1.054$ & 0.063 \\
\hline Tracheotomy & 0.668 & $0.166-2.681-$ & 0.569 & 0.225 & $0.050-1.015$ & 0.052 \\
\hline Time from illness onset hospitalization ( $>5$ days) & 6.849 & $5.208-9.009$ & $<0.001$ & 6.289 & $4.695-8.403$ & $<0.001$ \\
\hline Hospital stay (> 17 days) & 0.481 & $0.380-0.609$ & $<0.001$ & 0.411 & $0.316-0.533$ & $<0.001$ \\
\hline Time from illness onset to ICU admission (> 7 days) & 6.364 & $1.836-22.061$ & 0.027 & 8.030 & $1.740-37.057$ & $<0.001$ \\
\hline Time from illness onset to discharge (> 23 days) & 1.180 & $0.935-1.489$ & 0.163 & 0.995 & $0.772-1.281$ & 0.968 \\
\hline Time from illness onset to death (> 10 days) & 4.706 & $0.399-55.447$ & 0.218 & 4.731 & $0.314-71.265$ & 0.261 \\
\hline Time from hospitalization to ICU admission (> 4 days) & 0.857 & $0.243-3.024$ & 0.811 & 0.665 & $0.122-3.620$ & 0.637 \\
\hline Time from hospital admission to death (> 10 days) & 0.426 & $0.035-5.161$ & 0.502 & 0.155 & $0.007-3.694$ & 0.249 \\
\hline Duration of viral shedding (> 13 days) & 1.881 & $1.363-2.597$ & $<0.001$ & 1.640 & $1.153-2.333$ & 0.006 \\
\hline
\end{tabular}

${ }^{a}$ Adjusted for sex, age, smoking and Charlson Comorbidity Index ICU Intensive care unit, CI Confidence interval, OR Odds ratio

Subgroup analyses between Sichuan sub-cohorts with vs. without Wuhan-related exposure

There was almost no difference in clinical characteristics and outcomes between the two sub-cohorts with and without Wuhan-related exposure in Sichuan. Detailed information is provided in Supplementary Data (Tables S3, S4 and S5).

\section{Sichuan sub-cohort with Wuhan-related exposure vs. Wuhan cohort}

The differences in the clinical characteristics and outcomes between the Sichuan sub-cohort with Wuhan-related exposure and Wuhan cohort were similar to the differences between the Sichuan and Wuhan cohorts. The results are described in detail in the Supplementary Data (Tables S6, S7 and S8).

Table 4 Correlation of time from illness onset to hospitalization with systemic inflammation and features of organ dysfunction

\begin{tabular}{|c|c|c|c|c|c|c|c|c|}
\hline \multirow[t]{2}{*}{ Variables } & \multicolumn{2}{|c|}{ Model 1} & \multicolumn{2}{|c|}{ Model 2} & \multicolumn{2}{|c|}{ Model 3} & \multicolumn{2}{|c|}{ Model 4} \\
\hline & $r$ & $P$ value & $r$ & $P$ value & $r$ & $P$ value & $r$ & $P$ value \\
\hline White blood cell count, $\times 10^{9} / \mathrm{L}$ & 0.086 & 0.004 & 0.081 & 0.007 & 0.082 & 0.007 & 0.080 & 0.008 \\
\hline Neutrophil count, $\times 10^{9} / \mathrm{L}$ & 0.089 & 0.003 & 0.061 & 0.044 & 0.064 & 0.035 & 0.061 & 0.046 \\
\hline Lymphocyte count, $\times 10^{9} / \mathrm{L}$ & -0.026 & 0.391 & 0.027 & 0.380 & 0.025 & 0.414 & 0.032 & 0.293 \\
\hline Eosinophil count, $\times 10^{9} / \mathrm{L}$ & 0.116 & $<0.001$ & 0.119 & $<0.001$ & 0.118 & $<0.001$ & 0.127 & $<0.001$ \\
\hline Hemoglobin, g/L & -0.155 & $<0.001$ & -0.100 & 0.001 & -0.104 & 0.001 & -0.103 & 0.001 \\
\hline Platelet count, $\times 10^{9} / \mathrm{L}$ & 0.212 & $<0.001$ & 0.225 & $<0.001$ & 0.223 & $<0.001$ & 0.225 & $<0.001$ \\
\hline BUN, mmol/L & 0.100 & 0.002 & 0.038 & 0.247 & 0.040 & 0.215 & 0.039 & 0.226 \\
\hline Creatinine, $\mu \mathrm{mol} / \mathrm{L}$ & -0.094 & 0.003 & -0.117 & $<0.001$ & -0.141 & $<0.001$ & -0.141 & $<0.001$ \\
\hline Creatine kinase, U/L & -0.155 & $<0.001$ & -0.145 & $<0.001$ & -0.165 & $<0.001$ & -0.166 & $<0.001$ \\
\hline Albumin, $g / L$ & -0.263 & $<0.001$ & -0.200 & $<0.001$ & -0.194 & $<0.001$ & -0.192 & $<0.001$ \\
\hline APTT, s & -0.247 & $<0.001$ & -0.216 & $<0.001$ & -0.212 & $<0.001$ & -0.212 & $<0.001$ \\
\hline PT, s & -0.018 & 0.600 & -0.019 & 0.567 & -0.021 & 0.537 & -0.021 & 0.535 \\
\hline D-dimer, mg/L & 0.101 & 0.004 & 0.043 & 0.210 & 0.047 & 0.175 & 0.046 & 0.182 \\
\hline C-reactive protein, mg/L & 0.051 & 0.210 & 0.021 & 0.619 & 0.017 & 0.674 & 0.014 & 0.731 \\
\hline Procalcitonin, ng/mL & -0.093 & 0.019 & -0.123 & 0.002 & -0.130 & 0.001 & -0.135 & 0.001 \\
\hline IL-6, pg/mL & -0.094 & 0.407 & -0.155 & 0.171 & -0.144 & 0.211 & -0.144 & 0.215 \\
\hline
\end{tabular}

Model 1: Unadjusted; Model 2: Adjusted for age; Model 3: Adjusted for age, sex, smoking; Model 4: Adjusted for age, sex, smoking, and use steroid use APTT Activated partial thromboplastin time, PT Prothrombin time, BUN Blood urea nitrogen 


\section{Discussion}

To the best of our knowledge, there exists a paucity of information obtained from a comparative large-sample study on the differences in epidemiology, clinical characteristics and outcomes of patients with COVID-19 between the epicenter (Wuhan) and the peripheral areas of pandemic. This comparative study provides important insights. First, the outbreak and transmission of COVID19 within the region of Sichuan as the peripheral epidemic area has been well contained within 2 months through the use of traditional public health outbreak response tactics. Second, the Sichuan cohort is characterized by a higher incidence of upper airway symptoms, whereas the Wuhan cohort was older, had fewer lower airway symptoms and comorbidities, and had elevated pivotal systemic inflammation indicative of organ dysfunction as well as worse clinical outcomes independent of sex, age, smoking and comorbidities. Third, the subgroup analysis indicated that, within the Sichuan cohort, the patients with Wuhan-related exposure had similar clinical features and outcomes to those with nonWuhan-related exposure. Fourth, the Wuhan-related exposure patients in the Sichuan cohort had better clinical outcomes than those in the Wuhan cohort, although these two groups of patients had a similar Wuhanrelated exposure history.

As indicated in recently published studies [12], the COVID-19 patients in Wuhan, at the epicenter area of the epidemic, were older, had more co-existing conditions assessed by the CCI, had extended time from illness onset to hospitalization, and included more severely ill patients. However, the Sichuan cohort, as the peripheral area, had some characteristics features. First, there were fewer healthcare workers in the Sichuan cohort than in the Wuhan cohort, which could be at least partially explained by the insufficient implementation of precautions and the overwhelmed health system during the earlier stage of this outbreak in Wuhan. Second, intriguingly, there was a higher incidence of upper airway symptoms, rather than high incidence of lower airway symptoms in the Wuhan cohort at the epicenter epidemic, which was similar to the findings from exported cases in Singapore [18]. Accordingly, the exported patients from the epicenter were usually diagnosed with a "common cold" at the beginning of the COVID-19 outbreak. The different populations, the airway proliferation location, or the evolution of SARS-CoV-2 possibly could account for these differential symptoms [18-21]. Third, within the consecutively recruited cases in the Sichuan cohort as a well-defined population, the subgroup analyses indicated a higher proportion of males and older patients among the non-Wuhan-related exposure patients, which supported the theory of the propensity for SARS-CoV-2 infection in males and elders [9, 22, 23].
Recent studies from the USA and Italy have reported that a greater proportion of elderly and male COVID-19 patients would experience more critical illness [24, 25].

Until now no antiviral treatment for COVID-19 has proven effective, and supportive care is the mainstay of treatment is. Compared with the Wuhan cohort, the use of antibiotics (i.e. cephalosporin and quinolones) and glucocorticoids in the Sichuan cohort decreased by 26.4 and $16.1 \%$, respectively. These results could possibly be explained as follows. First, as indicated earlier, the expert panel drawn from the multidisciplinary team established by HCSP together developed and adjusted the treatment plan for severely or critically ill patients according to the interim guidance from the National Health Commission of China and the WHO across the 208 designated hospitals in Sichuan by using the $5 \mathrm{G}$ network every day. Accordingly, the use of systemic corticosteroids was strictly managed and they were not routinely administered for the treatment of COVID-19 patients. Second, the COVID-19 patients in the Wuhan cohort would actually be more severe or critically ill, which was supported by the increased use of supplemental oxygen in case of acute hypoxia. In addition, prone-position ventilation, physical rehabilitation and a variety of traditional Chinese medicines were used more often in Sichuan under the guidance of the expert panel; however, this aspect needs to be investigated further in randomized controlled trials $[7,8]$.

In terms of clinical outcomes, several important findings were identified in this study. An epidemic outbreak provided an opportunity to obtain important information, some of which were associated with a limited window of opportunity. This study showed that there was a delay from illness onset to hospitalization in the Wuhan cohort, which might be an important risk factor for the progression of COVID-19. Multivariate regression analysis showed that the time from illness onset to hospitalization was significantly associated with mortality and ICU admission, which suggested some important implications with regard to the pathogenesis of SARS$\mathrm{CoV}-2$ and may provide insights into a unique window of opportunity for intervention [7]. Liang et al. [12] recently found that Wuhan-related exposure patients have worse clinical outcomes compared with the nonWuhan-related exposure cases; they attributed the attenuated disease to the onward transmission of SARS-CoV2. In fact, this is paradoxical to the findings reported from Liang et al.'s study [12] because the relationship between Wuhan-related exposure and prognosis disappeared after adjusting for confounders. Our study firstly found that COVID-19 patients in the Wuhan cohort had worse clinical outcomes including case fatality rate, ICU admission, and duration of virus shedding, independent of sex, age, smoking, comorbidities, and even time from 
illness onset to hospitalization. The severity of COVID19 and the shortage of medical resources would partly account for these worse outcomes. For example, during an earlier stage of the outbreak, some patients would not have received sufficient oxygen support because of insufficient oxygen pressure.

The duration of infectious virus replication is an important factor in assessing the risk of transmission and for guiding decisions on the isolation of patients; however, the duration of SARS-CoV-2 RNA detection has not been well explored. Our study found that the $\mathrm{Wu}$ han cohort in the epicenter area had the prolonged virus shedding, which may contribute to the disease severity and clinical course [26, 27]. Furthermore, we found for the first time that the duration of virus shedding was independently associated with age and time from illness onset to hospitalization. Our findings are supported by those of other studies. Liu et al. [28] found that the viral load in severe cases was higher than that of mild cases, which had early viral shedding. Wolfel et al. [29] found that virus shedding in the upper airway, which is the location of mild COVID-19, was very high during the first week of symptoms, whereas shedding of viral RNA from sputum derived from the lower airway, which is the region of general to critical illness in COVID-19, outlasts the disappearance of symptoms. Xu et al. [30] found that elderly patients had prolonged virial shedding, but the correlation of age with the duration of viral shedding disappeared after adjusting for confounders, although this might be partly attributed to the small sample size.

As the pandemic evolves, mutations and natural selection of SARS-CoV-2 inevitably occur, although this virus a lower mutation rate than that of other RNA viruses [31]. The China National Center for Bioinformation aligned 77,801 genome sequences of SARS-CoV-2 that were detected globally and identified a total of 15,018 mutations [32]. Studies have shown that mutations play an important role in the virulence and infectivity of SARS-CoV-2, although no significant association was found between mutations and outcomes pertaining to hospitalization or death [33-35]. Thus, it is unclear whether the different clinical outcomes of patients with COVID-19 between the epicenter and peripheral areas affected by the pandemic are due to mutations in SARS-CoV-2.

This large-sample comparative study provides informative insights into the differences in epidemiology, clinical characteristics and outcomes of patients with COVID-19 between the epicenter (Wuhan) and peripheral (Sichuan) areas of the pandemic. However, there are several limitations that need to be addressed. First, due to the retrospective study design, data generation was clinically driven, and not all laboratory data were available for all patients. Accordingly, the missing data for some patients may have biased the findings. Second, the Sichuan cohort, which represented the peripheral area of the COVID-19 pandemic, was incomplete although consecutive patients accounting for $88.1 \%$ of total cases with COVID-19 were recruited from 41 designated hospitals in Sichuan. Third, we did not analyze the genetic diversity of virus strains and the evolutionary history, which might well explain the differences between the epicenter and peripheral areas affected by the pandemic.

\section{Conclusions}

This comparative study found that there were significant differences in the epidemiology, clinical characteristics, and outcomes of patients with COVID-19 between the epicenter and peripheral areas affected by the pandemic. The worse outcomes in the epicenter could be partly explained by the overwhelming of health systems and the delayed time from illness onset to hospitalization. This was associated with elevated systemic inflammation, organ dysfunction and prolonged duration of virus shedding, independent of sex, age, smoking and comorbidities. This has potential implications that are of clinical relevance in interventions for COVID-19. The data suggests that urgent or early supportive care would achieve improved clinical outcomes, leading to a lower death rate although no proven effective therapies currently exist. No differences were found in the epidemiology, clinical characteristics, and outcomes between the first generation and secondary generation of patients in the peripheral area of pandemic. Biological differences accounting for the differences between the Wuhan-related exposure patients in the Sichuan cohort and Wuhan cohort need to be further investigated.

\section{Supplementary Information}

The online version contains supplementary material available at https://doi. org/10.1186/s12879-020-05728-7.

\footnotetext{
Additional file 1: Table S1. Detailed comorbidities of patients in Sichuan and Wuhan cohorts. Table S2. Regression analysis of the risk factors for death, ICU admission and mechanical ventilation in all patients from the Sichuan and Wuhan cohorts. Table S3. Demographics and clinical characteristics of patients in Sichuan sub-cohorts with vs. without Wuhan-related exposure. Table S4. Outcomes of patients in Sichuan sub-cohorts with vs. without Wuhan-related exposure. Table S5. Risk of adverse outcomes in Sichuan sub-cohorts with vs. without Wuhan-related exposure. Table S6. Demographics and clinical characteristics of patients in Sichuan sub-cohort with Wuhan-related exposure vs. Wuhan cohort. Table S7. Outcomes in Sichuan sub-cohort with Wuhan-related exposure vs. Wuhan cohort. Table S8. Risk of adverse outcomes in Sichuan subcohort with Wuhan-related exposure vs. the Wuhan cohort
}

\section{Abbreviations}

aOR: Adjusted odds ratio; CCl: Charlson comorbidity index; Cl: Confidence interval; COVID-19: Coronavirus disease 2019; HCSP: The Health Commission of Sichuan Province; ISARIC: International Severe Acute Respiratory and Emerging Infection Consortium; OR: Odds ratio; SARS-CoV-2: Severe acute respiratory syndrome coronavirus 2; WHO: World health organization 


\section{Acknowledgments}

We would like to thank all the patients who donate their data for this analysis and all medical staffs working in the front line for taking care of the patients. We show thanks to Dr. Xuan Zhang, PhD, Dr. Xin Zhang, MD, Dr. Ting Wang, MD, and Dr. Ji Wang, MD, for their statistical analyses and editing work. We are indebted to the coordination of Ms. Jun Hua Li from the Health Commission of Sichuan Province, and Mr. Nian Li and Ms. Bei Bai from West China Hospital, Sichuan University, who greatly facilitate the collection of patients' data.

We appreciate the expert panel from multiple disciplines team as follows: (1) Respiratory and Critical Care Medicine: Zong An Liang, Feng Min Luo, Chun Tao Liu, Hong Fan, Dan Liu, He Yu at West China Hospital, Sichuan University, Yang Yang from Sichuan Academy of Medical Sciences, Sichuan Provincial People's Hospital,Song Ping Wang, Wen Jun Wang from Affiliated Hospital of Southwest Medical University, Xiao Ju Chen, Li Jiang at Affiliated Hospital of North Sichuan Medical College, Zhen Liang Xiao at General Hospital of Western War Zone, Qing Song Huang, Yan Dong at Affiliated Hospital of Chengdu University of Traditional Chinese Medicine, De Hai Yu from the Second Traditional Chinese Medicine Hospital of Sichuan, Xiao Hui Zhang from Sichuan Integrative Medicine Hospital, Su Hua Ao from Affiliated Traditional Chinese Medicine Hospital of Southwest Medical University, Yong Sheng Wang from The first People's Hospital of Chengdu, Guo Ping Li from The Third People's Hospital of Chengdu, Ya Lin Liu, Gui Hui Wu at Public Health Clinical Center of Chengdu, Wei Guo Xu from Mianyang Central Hospital, Zheng Guang He from Suining Central Hospital; (2) Nosocomial infection: Zhi Yong Zong, Wei Jia Yin at West China Hospital, Sichuan University, Jian Jun Deng at The Second West China Hospital, Sichuan University, Jia Yu Wu at Sichuan Provincial Peoples Hospital, Rong Li Wang, Jiao Xia at The affiliated hospital of Southwest Medical University, Zong Ying Jing at The TSC hospital of Southwest Medical University, Yong Fang Liu, Bi Feng Xu at The affiliated hospital of North Sichuan Medical College, Zhang Chen at The General Hospital of Western theater Command, Wen Sheng Zhang at Affiliated Hospital of Chengdu University of TCM, Hua Guo at Chengdu Third People's Hospital, Ping Chen, Xiao Fei Duan at Public Health and Clinical Center of Chengdu, Chun Hua Ma at Mianyang 404 Hospital; (3) Radiology: Bin Song, Hong Li Bai, Jian Qun Yu at West China Hospital, Sichuan University, Jin Jiang at Sichuan Provincial Peoples Hospital, Jian Shu, Fu Gang Hang at The affiliated hospital of Southwest Medical University, Tian Wu Chen, Qiong Hui Zhao at The affiliated hospital of North Sichuan Medical College, Rui Jiang at The General Hospital of Western theater Command, Ming Guo Xie at Affiliated Hospital of Chengdu University of TCM, Bin Yang at The TSC hospital of Southwest Medical University, Na Zhang, Xin Wei Wang at Public Health and Clinical Center of Chengdu, Qi Yu Liu at Mianyang Central Hospital; (4) Department of Laboratory Medicine: Kang Mei, Xie Yi at West China Hospital, Sichuan University, Jiang Li, Yu Hua from Sichuan Provincial People's Hospital,Liu Jin Bo, Li Bao Lin from Affiliated Hospital of Southwest Medical University, Zhang Guo Yuan, Huang Yi Shan from Affiliated Hospital of North Sichuan Medical College, Hu Zong Hai, Dan Gang from the General Hospital of Western Theater Command, Xiong Da Qian from Chengdu University of Traditional Chinese Medicine, Guo Yong Can from Southwest Medical University of Traditional Chinese Medicine, Li Qing Feng, Cao Ling from the Public Health Clinical Center of Chengdu; (5) Diagnosis and Treatment of Infectious Diseases: Tang Hong, Feng Ping, Lv Xiao Ju, Bai Lang at West China Hospital, Sichuan University, Yang Xing Xiang from Sichuan Provincial People's Hospital, Huang Yong Mao, Huang Fu Li from Affiliated Hospital of Southwest Medical University, Xiong Liang Shi, Liu Feng Jun from Affiliated Hospital of North Sichuan Medical College, Zhang Ling,Zeng Yi Lan from the General Hospital of Western Theater Command, He Sheng Hua from the Public Health Clinical Center of Chengdu;(6) Critical Care Medicine: Yan Kang, Xiao Dong Jin, Zhong Wei Zhang at West China Hospital, Sichuan University, Xiao Bo Huang from Provincial People's Hospital, Xian Ying Lei, Chang Xue Wu at the Affiliated Hospital of Southwest Medical University, Ying Ge, Da Qing Wang at the Affiliated Hospital of North Sichuan Medical College, Fu Xiang Li, Zhang Wang at the General Hospital of Western Theater Command, Pei Yang Gao from Affiliated Hospital of Chengdu University of Traditional Chinese Medicine, Xiao Bin Li from Affiliated Hospital of Traditional Chinese Medicine of Southwest Medical University, Yun Feng Chen from Chengdu Integrated Traditional Chinese Medicine \& Western Medicine Hospital, Chuan Zhang from Chengdu Third People's Hospital, Hong Chen, Hong De Chen, Ling Zhang at Chengdu Public Health Clinical Medical Center; (7) Emergency treatment: Jun Zeng, Ping Zhou, Xiao Mei Wang at Provincial People's Hospital, Yu Cao from West China Hospital, Sichuan University, Wu Zhong, Ying Liu at the Affiliated Hospital of Southwest Medical University, Shi Ping Liu, Zhi Jiang at the Affiliated Hospital of North Sichuan Medical College, Gui Sen Xu from the General Hospital of Western Theater Command, Yun Lu, Xiao Yun Zhang at Affiliated Hospital of Chengdu University of Traditional Chinese Medicine, Zhi Hong Zhang from Affiliated Hospital of Traditional Chinese Medicine of Southwest Medical University, Yu Fei Hou from Chengdu Emergency Command Center, Wen Jun Li, Bo Leng at Chengdu Public Health Clinical Medical Center; (8) Traditional Chinese Medicine: Shao Hong Chen, Cheng Shi He,Xiao Yu Hu, Kun Yang at Affiliated Hospital of Chengdu University of Traditional Chinese Medicine, Gang Suo from Sichuan Provincial Orthopedics Hospital.

We also show thanks to Li Yan A, Ling Chen, Jing Yu Cui, Ke Deng, Su Jun Deng, Xue Mei Fang, Min Feng, Xiao Min Fu, Yu Yu Han, Jia Xin Hou, Yue Hong Hu, Dan Huang, Qiao Yun Huang, Min Li, Ren Jiao Li, Xiao Qian Li, Yu Qi Li, Jun Zhe Liao, Ping Lin, Zhi Lin, Dan Liu, Jia Liu, Lei Liu, Sha Liu, Shi Yao Liu, Zhi Hao Liu, Ya Lan Luo, Zhi Wen Ma, Min Qi, Xing Yu Qiu, Fang Yu Shi, Tao Lin Qin, Xiao Ju Tang, Rui Tian, Xin Tian, Dan Wang, Ji Wang, Qin Wang, Ting Wang, Yi Wei Wang, Yu Wang, Jia Hao Wu, Wen Wen Wu, Lin Rui Xu, Zhuan Hua Yang, Dong Fan Ye, Yuan Ye, Ze Lin Yin, Yu Lai Yuan, Jing Xuan Zhang, Liang Liang Zhang, Xin Zhang, Ya Li Zhang, Yu Yang Zhang, Yu Chen Zhang, Li Chun Zhong, Qiao Zhou, Yu Yan Zhou, Wen Juan Zhu, Wen Jun Zhu, Ying Ying Zhu, En Qiang Chen, Ming Jin Dai, Hui Gao, Hong Bo Jia, Yang Li, Min Tao Lin, Qi Liu, Lu Lu, Ruo Ting Men, Zhi Ming Ni, Da Nan Pan, Yuan Ya Qin, Tian Rui Ren, Si Si Shen, Yan Jun Si, Jia Jia Song, Xing Bo Song, Li Yi Wang, Wen Xiu Wang, Yong Wang, Xi Zhan Weng, Qin Wu, Yu Jie Wu, Xian Xing, Rong Fang Yan, Zhi Qing Yan, Lan Yang, Le Tian Yang, Ying Ying Yang, Jia Yu Ye, Ling Zhang, Qi Zhang, Sheng Zhang, Ying Teng Zhang, Ying Ying Zhang, Yu Wei Zhang, Han Jiang Zeng for their dedication to data entry and verification.

We also show thanks to hospitals who recruited the included cases as following: West China Hospital of Sichuan University, The Public Health Clinical Center of Chengdu, People's hospital of Jianyang, Pidu District People's Hospital of Chengdu, Zigong First People's Hospital, Jintang County First People's Hospital, People's Hospital of Deyang City, Sichuan Mianyang 404 Hospital, The Third People's Hospital of Guangyuan, The Fourth People's Hospital of Panzhihua, Luzhou Infectious Diseases Hospital, Suining Central Hospital, The First People's Hospital of Neijiang, Neijiang Second Municipal People's Hospital, People's Hospital of Leshan, Affiliated Hospital of North Sichuan Medical College, The Second People's Hospital of Yibin, Linshui County Peoples's Hospital of Guangan, Yuechi County Peoples's Hospital, Guang'an People's Hospital (West China-Guang'an Hospital, Sichuan University), People's Hospital of Huaying, Dazhou Central Hospital, Bazhong Central Hospital, Third Branch of Yaan People's Hospital (Hospital of Infectious Diseases), Meishan City People's Hospital, The First People's Hospital of Ziyang, The First People's Hospital of Liangshan Yi Autonomous Prefecture, Ganluo County People's Hospital of Liangshan Yi Autonomous Prefecture, People's Hospital of Aba Tibetan and Qiang Autonomous Prefecture, Ganzi Tibetan Autonomous Prefecture People's Hospital, Nanchong Central Hospital, Pengan County People's Hospital, People's Hospital of Langzhong, Nanbu County People's Hospital, The Fifth People's Hospital of Nanchong, Yilong County People's Hospital, Yingshan County People's Hospital, Pinchang County People's Hospital, Tongjiang County People's Hospital, Medical Center Hospital of Qionglai, Jiangyou Infectious Diseases Hospital.

\section{Authors' contributions}

GW, FML, DL, JSL, NX, JFL, ZAL, WML had roles in the study design, data analysis, data interpretation, and writing of the manuscript. YW, HC, PWT, TF, LT, HY, LW, MF, ZN, BW, ZFS, XLW, HJW, XT, MX, XYL, BL, CJ, JX, JS had roles in the collection, processing, cleaning, and interpretation of data. All authors read and approved the final manuscript.

\section{Funding}

This study was supported by the Sichuan Provincial Program for Diagnostic and Treatment of COVID-19 (No. 2020 YFS002 and 2020YFS005), and the National Key Development Plan for Precision Medicine Research (2017YFC091004), and the National Natural Science Foundation of China (No. 81920108002). Funding sources played no role in the study design, data analysis, decision to publish, or preparation of the manuscript. 


\section{Availability of data and materials}

The datasets used and/or analysed during the current study are available from the corresponding author on reasonable request.

\section{Ethics approval and consent to participate}

The Biological and Medical Ethics Committee of West China Hospital (approval number: 2020-304 and 2020-126) and the Ethic Committee of Renmin Hospital of Wuhan University (approval number: WDRY2020-K068) granted ethical approval for this study. Administrative permission to access the raw data was granted by administrators of each hospital. Written informed consent from each participant was waived for design of a retrospective study. The data used in this study was anonymised before its use.

\section{Consent for publication}

Not applicable.

\section{Competing interests}

The authors declare that they have no competing interests.

\section{Author details}

${ }^{1}$ Department of Respiratory and Critical Care Medicine, Clinical Research Center for Respiratory Disease, West China Hospital, Sichuan University, Chengdu 610041, Sichuan, China. ${ }^{2}$ Laboratory of Pulmonary Immunology and Inflammation, Frontiers Science Center for Disease-related Molecular Network, Sichuan University, Chengdu 610041, Sichuan, China. ${ }^{3}$ Department of Gastrointestinal Surgery, Renmin Hospital of Wuhan University, Wuhan 430060, Hubei, China. ${ }^{4}$ Department of Critical Care Medicine, Public Health Clinical Center of Chengdu, Chengdu 610061, Sichuan, China. ${ }^{5}$ Department of Integrated Traditional Chinese and Western Medicine, West China Hospital, Sichuan University, Chengdu 610041, Sichuan, China. ${ }^{6}$ Department of Respiratory Medicine, Dazhou Central Hospital, Dazhou 635000, Sichuan, China. ${ }^{7}$ Department of Critical Care Medicine, Affiliated Hospital of Southwest Medical University, Luzhou 646000, Sichuan, China. ${ }^{8}$ Mianyang 404 Hospital, Mianyang 621000, Sichuan, China. ${ }^{9}$ Department of Critical Care Medicine, Mianyang Central Hospital, Mianyang 621000, Sichuan, China. ${ }^{10}$ Department of Respiratory and Critical Care Medicine, People's Hospital of Ganzi Prefecture, Ganzi 626700, Sichuan, China. "'Department of Critical Care Medicine, Nanchong Central Hospital, Nanchong 637000, Sichuan, China. ${ }^{12}$ Department of Neurology, Union Hospital, Tongji Medical College, Huazhong University of Science and Technology, Wuhan 430022, Hubei, China. ${ }^{13}$ Wuhan Red Cross Hospital, Wuhan 430015, Hubei, China.

Received: 30 July 2020 Accepted: 21 December 2020 Published online: 24 February 2021

\section{References}

1. Wenjie T, Xiang Z, Xuejun M, Wenling W, Peihua N, Wenbo X, George FG, Guizhen W. A novel coronavirus genome identified in a cluster of pneumonia cases - Wuhan, China 2019-2020. China CDC Weekly. 2020; 2(4):61-2.

2. Huang C, Wang Y, Li X, Ren L, Zhao J, Hu Y, Zhang L, Fan G, Xu J, Gu X, et al. Clinical features of patients infected with 2019 novel coronavirus in Wuhan, China. Lancet. 2020;395(10223):497-506.

3. Wang D, Hu B, Hu C, Zhu F, Liu X, Zhang J, Wang B, Xiang H, Cheng Z, Xiong $Y$, et al. Clinical characteristics of 138 hospitalized patients with 2019 novel coronavirus-infected pneumonia in Wuhan, China. JAMA. 2020; 323(11):1061-9.

4. Chen N, Zhou M, Dong X, Qu J, Gong F, Han Y, Qiu Y, Wang J, Liu Y, Wei Y, et al. Epidemiological and clinical characteristics of 99 cases of 2019 novel coronavirus pneumonia in Wuhan, China: a descriptive study. Lancet. 2020; 395(10223):507-13

5. Zeng Y, Li DJ, Zong ZY, Jiang Y. on behalf of Sichuan Medical Treatment Expert Group for Covid-19: The emergency prevention and control guidelines for Covid-19 in medical institutions of Sichuan province. In: Sichuan Science and Technology Press; 2020.

6. Brooks SK, Webster RK, Smith LE, Woodland L, Wessely S, Greenberg N, Rubin GJ. The psychological impact of quarantine and how to reduce it: rapid review of the evidence. Lancet. 2020;395(10227):912-20.

7. Fauci AS, Lane HC, Redfield RR. Covid-19 - navigating the uncharted. N Engl J Med. 2020;382(13):1268-9.
8. Sanders JM, Monogue ML, Jodlowski TZ, Cutrell JB. Pharmacologic treatments for coronavirus disease 2019 (COVID-19): a review. JAMA. 2020 323(18):1824-36.

9. Cai Q, Huang D, Ou P, Yu H, Zhu Z, Xia Z, Su Y, Ma Z, Zhang Y, Li Z, et al. COVID-19 in a designated infectious diseases hospital outside Hubei Province, China. Allergy. 2020;75(7):1742-52.

10. $X u X W, W u X X$, Jiang $X G$, Xu KJ, Ying $L J, M a C L$, Li SB, Wang HY, Zhang S, Gao HN, et al. Clinical findings in a group of patients infected with the 2019 novel coronavirus (SARS-Cov-2) outside of Wuhan, China: retrospective case series. BMJ. 2020;368:m606.

11. Tian S, Hu N, Lou J, Chen K, Kang X, Xiang Z, Chen H, Wang D, Liu N, Liu D, et al. Characteristics of COVID-19 infection in Beijing. J Inf Secur. 2020;80(4):401-6.

12. Liang WH, Guan WJ, Li CC, Li YM, Liang HR, Zhao Y, Liu XQ, Sang L, Chen $\mathrm{RC}$, Tang $\mathrm{CL}$, et al. Clinical characteristics and outcomes of hospitalised patients with COVID-19 treated in Hubei (epicenter) and outside Hubei (non-epicenter): a Nationwide analysis of China. Eur Respir J. 2020;55(6).

13. WHO. Novel Coronavirus-China. 2020. https://www.who.int/csr/don/12january-2020-novel-coronavirus-china/en/2020. Accessed May 82020.

14. Wei JF, Huang FY, Xiong TY, Liu Q, Chen H, Wang H, Huang H, Luo YC, Zhou X, Liu ZY, et al. Acute myocardial injury is common in patients with COVID-19 and impairs their prognosis. Heart. 2020;106(15):1154-9.

15. Xiong W, Mu J, Guo J, Lu L, Liu D, Luo J, Li N, Liu J, Yang D, Gao H, et al. New onset neurologic events in people with COVID-19 infection in three regions in China. Neurology. 2020;95(11):e1479-87.

16. Xiong TY, Huang FY, Liu Q, Peng Y, Xu YN, Wei JF, Li N, Bai B, Li JH, Prendergast $B$, et al. Hypertension is a risk factor for adverse outcomes in patients with coronavirus disease 2019: A cohort study. Ann Med. 2020:1-20.

17. Charlson ME, Pompei $P$, Ales $K L$, Mackenzie CR. A new method of classifying prognostic comorbidity in longitudinal studies: development and validation. J Chronic Dis. 1987;40(5):373-83.

18. Young BE, Ong SWX, Kalimuddin S, Low JG, Tan SY, Loh J, Ng OT, Marimuthu K, Ang LW, Mak TM, et al. Epidemiologic features and clinical course of patients infected with SARS-CoV-2 in Singapore. JAMA. 2020; 323(15):1488-94.

19. Li X, Wang W, Zhao X, Zai J, Zhao Q, Li Y, Chaillon A. Transmission dynamics and evolutionary history of 2019-nCoV. J Med Virol. 2020;92(5):501-11.

20. Zhang J, Litvinova M, Wang W, Wang Y, Deng X, Chen X, Li M, Zheng W, Yi $L$, Chen $X$, et al. Evolving epidemiology and transmission dynamics of coronavirus disease 2019 outside Hubei province, China: a descriptive and modelling study. Lancet Infect Dis. 2020;20(7):793-802.

21. Rubens JH, Karakousis PC, Jain SK. Stability and viability of SARS-CoV-2. N Engl J Med. 2020;382(20):1962-3.

22. Liu Y, Mao B, Liang S, Yang JW, Lu HW, Chai YH, Wang L, Zhang L, Li QH, Zhao $L$ et al. Association between ages and clinical characteristics and outcomes of coronavirus disease 2019. Eur Respir J. 2020;55(5).

23. Zhou F, Yu T, Du R, Fan G, Liu Y, Liu Z, Xiang J, Wang Y, Song B, Gu X, et al. Clinical course and risk factors for mortality of adult inpatients with COVID19 in Wuhan, China: a retrospective cohort study. Lancet. 2020;395(10229): 1054-62.

24. Richardson S, Hirsch JS, Narasimhan M, Crawford JM, McGinn T, Davidson KW, the Northwell C-RC, Barnaby DP, Becker LB, Chelico JD, et al. Presenting characteristics, comorbidities, and outcomes among 5700 patients hospitalized with COVID-19 in the New York City area. JAMA. 2020;323(20): 2052-9.

25. Grasselli G, Zangrillo A, Zanella A, Antonelli M, Cabrini L, Castelli A, Cereda D, Coluccello A, Foti G, Fumagalli R, et al. Baseline characteristics and outcomes of 1591 patients infected with SARS-CoV-2 admitted to ICUs of the Lombardy region, Italy. JAMA. 2020;323(16):1574-81.

26. Liu Y, Yan LM, Wan L, Xiang TX, Le A, Liu JM, Peiris M, Poon LLM, Zhang W. Viral dynamics in mild and severe cases of COVID-19. Lancet Infect Dis. 2020;20(6):656-7.

27. Zhou B, She J, Wang Y, Ma X. The duration of viral shedding of discharged patients with severe COVID-19. Clin Infect Dis. 20;71(16):2240-2.

28. Liu Y, Eggo RM, Kucharski AJ. Secondary attack rate and superspreading events for SARS-CoV-2. Lancet. 2020;395(10227):e47.

29. Wolfel R, Corman VM, Guggemos W, Seilmaier M, Zange S, Muller MA, Niemeyer D, Jones TC, Vollmar P, Rothe C, et al. Virological assessment of hospitalized patients with COVID-2019. Nature. 2020;581(7809):465-9.

30. Xu K, Chen Y, Yuan J, Yi P, Ding C, Wu W, Li Y, Ni Q, Zou R, Li X, et al. Factors associated with prolonged viral RNA shedding in patients with COVID-19. Clin Infect Dis. 2020;71(15):799-806. 
31. Zhao Z, Sokhansanj BA, Malhotra C, Zheng K, Rosen GL. Genetic grouping of SARS-CoV-2 coronavirus sequences using informative subtype markers for pandemic spread visualization. PLoS Comput Biol. 2020;16(9):e1008269.

32. Hu B, Guo H, Zhou P, Shi ZL. Characteristics of SARS-CoV-2 and COVID-19. Nat Rev Microbiol. 2020. p. 1-4.

33. Chen J, Wang R, Wang M, Wei GW. Mutations strengthened SARS-CoV-2 infectivity. J Mol Biol. 2020;432(19):5212-26.

34. Korber B, Fischer WM, Gnanakaran S, Yoon H, Theiler J, Abfalterer W, Hengartner N, Giorgi EE, Bhattacharya T, Foley B, et al. Tracking changes in SARS-CoV-2 spike: evidence that D614G increases infectivity of the COVID19 virus. Cell. 2020;182(4):812-27 e819.

35. Nakamichi K, Shen IZ, Lee CS, Lee AY, Roberts EA, Simonson PD, Roychoudhury P, Andriesen JG, Randhawa AK, Mathias PC, et al. Outcomes associated with SARS-CoV-2 viral clades in COVID-19. medRxiv. 2020.

\section{Publisher's Note}

Springer Nature remains neutral with regard to jurisdictional claims in published maps and institutional affiliations.

Ready to submit your research? Choose BMC and benefit from:

- fast, convenient online submission

- thorough peer review by experienced researchers in your field

- rapid publication on acceptance

- support for research data, including large and complex data types

- gold Open Access which fosters wider collaboration and increased citations

- maximum visibility for your research: over $100 \mathrm{M}$ website views per year

At $\mathrm{BMC}$, research is always in progress.

Learn more biomedcentral.com/submissions 